\title{
A Model for a Standardized and Sustainable Pediatric Anesthesia-Intensive Care Unit Hand-Off Process
}

\author{
Priti G. Dalal ${ }^{1, *}$, Theodore J. Cios ${ }^{1}$, Theodore K. M. DeMartini ${ }^{2}$, Amit A. Prasad ${ }^{1}$, \\ Meghan C. Whitley ${ }^{1}$, Joseph B. Clark ${ }^{3}$, Leon Lin ${ }^{4}$, Dennis J. Mujsce ${ }^{5}$ and Robert E. Cilley ${ }^{6}$ \\ 1 Departments of Anesthesiology and Peri-Operative Medicine, \\ Penn State Health Milton S Hershey Medical Center, Penn State Health Children's Hospital, \\ Hershey, PA 17033, USA; tcios@pennstatehealth.psu.edu (T.J.C.); aprasad@pennstatehealth.psu.edu (A.A.P.); \\ mwhitley@pennstatehealth.psu.edu (M.C.W.) \\ 2 Division of Pediatric Critical Care, Department of Pediatrics, Penn State Health Children's Hospital, \\ Hershey, PA 17033, USA; tdemartini@pennstatehealth.psu.edu \\ 3 Division of Pediatric Cardiac Surgery, Department of Pediatrics, Penn State Health Children's Hospital, \\ Hershey, PA 17033, USA; jclark7@pennstatehealth.psu.edu \\ 4 Department of Emergency Medicine, Ohio State Universirty, Columbus, OH 43210, USA; \\ Lin.leon02@gmail.com \\ 5 Division of Newborn Medicine, Department of Pediatrics, Penn State Health Children's Hospital, \\ Hershey, PA 17033, USA; dmujsce@pennstatehealth.psu.edu \\ 6 Division of Pediatric Surgery, Department of Surgey, Penn State Health Children's Hospital, \\ Hershey, PA 17033, USA; rcilley@pennstatehealth.psu.edu \\ * Correspondence: pdalal@pennstatehealth.psu.edu
}

Received: 4 August 2020; Accepted: 1 September 2020; Published: 3 September 2020

check for updates

\begin{abstract}
Background and Objectives: The hand-off process between pediatric anesthesia and intensive care unit (ICU) teams involves the exchange of patient health information and plays a major role in reducing errors and increasing staff satisfaction. Our objectives were to (1) standardize the hand-off process in children's ICUs, and (2) evaluate the provider satisfaction, efficiency and sustainability of the improved hand-off process. Methods: Following multidisciplinary discussions, the hand-off process was standardized for transfers of care between anesthesia-ICU teams. A pre-implementation and two post-implementation (6 months, $>2$ years) staff satisfaction surveys and audits were conducted to evaluate the success, quality and sustainability of the hand-off process. Results: There was no difference in the time spent during the sign out process following standardization-median 5 min for pre-implementation versus 5 and 6 min for post-implementation at six months and $>2$ years, respectively. There was a significant decrease in the number of missed items (airway/ventilation, venous access, medications, and laboratory values pertinent events) post-implementation compared to pre-implementation $(p \leq 0.001)$. In the $>2$ years follow-up survey, $49.2 \%$ of providers felt that the hand-off could be improved versus $78.4 \%$ in pre-implementation and $54.2 \%$ in the six-month survey $(p<0.001)$. Conclusion: A standardized interactive hand-off improves the efficiency and staff satisfaction, with a decreased rate of missed information at the cost of no additional time.
\end{abstract}

Keywords: patient hand-off; intensive care; children; anesthesia

\section{Introduction}

Patient "sign-out" or "hand-off" is a critical part of the provision of medical care. This is particularly important with respect to critically ill patients, especially children. A hand-off is defined by The Joint Commission as the "real-time process of passing patient-specific information from one caregiver to another or from one team of caregivers to another for the purpose of ensuring the continuity of patient's 
care" [1]. The hand-off process involves the exchange of patient health information, as well as the transfer of patient care and responsibility. A detailed and pertinent hand-off also plays a major role in reducing errors [2], improving outcomes, and increasing staff satisfaction [3]. Studies have shown that intensive care units have a higher rate of medical errors compared to other units in the hospital $[4,5]$. While there is some literature surrounding the use of checklists and protocols for these hand-off processes with respect to the transition of care from the operating room (OR) to the intensive care unit (ICU) [6], there is not much literature on the transition of care from the ICU to the OR. Further, there is sparse data on the staff satisfaction, sustainability and effectiveness of a standardized hand-off process.

In our children's hospital, we identified the variability and lack of a formal hand-off process during OR-ICU and ICU-OR hand-off. We identified anecdotally missed critical information such as failed extubation, difficult intubation, medications administered, abnormal laboratory values (electrolyte abnormalities, coagulation abnormalities, etc.) that could potentially have led to errors in patient care, as well as overall provider dissatisfaction based on anecdotes from providers participating in the hand-off process. As a result, stake holders from anesthesia, surgery and ICU teams (intensivist, nurse, respiratory therapist) of the children's hospital met to discuss this as a quality improvement (QI) project. The aims of our QI project were to (1) standardize the process of anesthesia-ICU hand-off communication in children's ICUs and (2) evaluate provider satisfaction, efficiency and sustainability of a standardized hand-off process after the implementation of the process change.

\section{Materials and Methods}

The Institutional Review Board (IRB) waived the requirement for a review of this QI project.

\subsection{Setting}

This QI project was implemented at our children's hospital, which is an academic tertiary care center and American College of Surgeons Level 1 verified Children's Surgical Center. The 75-bed children's hospital is a part of the main university hospital. It is a dedicated stand-alone building connected to the adult hospital via a bridge. Our children's hospital encompasses two ICUs, i.e., a 42-bed neonatal intensive care unit (NICU) and an 18-bed pediatric intensive care unit (PICU). The anesthesia team, along with the surgical team, is involved with the transport of critically ill children from the ICU to the OR (Exit ICU) and from the OR to the ICU (Entry ICU). Hence, the transition of care of critically ill children occurs between multiple teams.

\subsection{Planning the Intervention}

Prior to the study, there was variability in the hand-off process with a potential for miscommunication, loss of information and provider dissatisfaction. There was a lack of clarity with regard to the start and end of the existent hand-off process, and the point when the onward clinical care responsibility was assumed by the receiving team.

A multidisciplinary team of experts met to discuss the existing hand-off process during the transition of care of children from the NICU and PICU. The team included stakeholders from anesthesiology, NICU, PICU, surgery, respiratory therapists and nursing staff. A baseline pre-implementation survey (Survey 1) was conducted in order to determine the provider perceptions of the current practice of hand-off and need for any improvements. It was identified that no standardized hand-off process or tool existed. Following multiple meetings and discussions, the team identified various problems and factors contributing to the variability in the hand-off process. Please refer to the fishbone diagram in Figure 1 for details. 


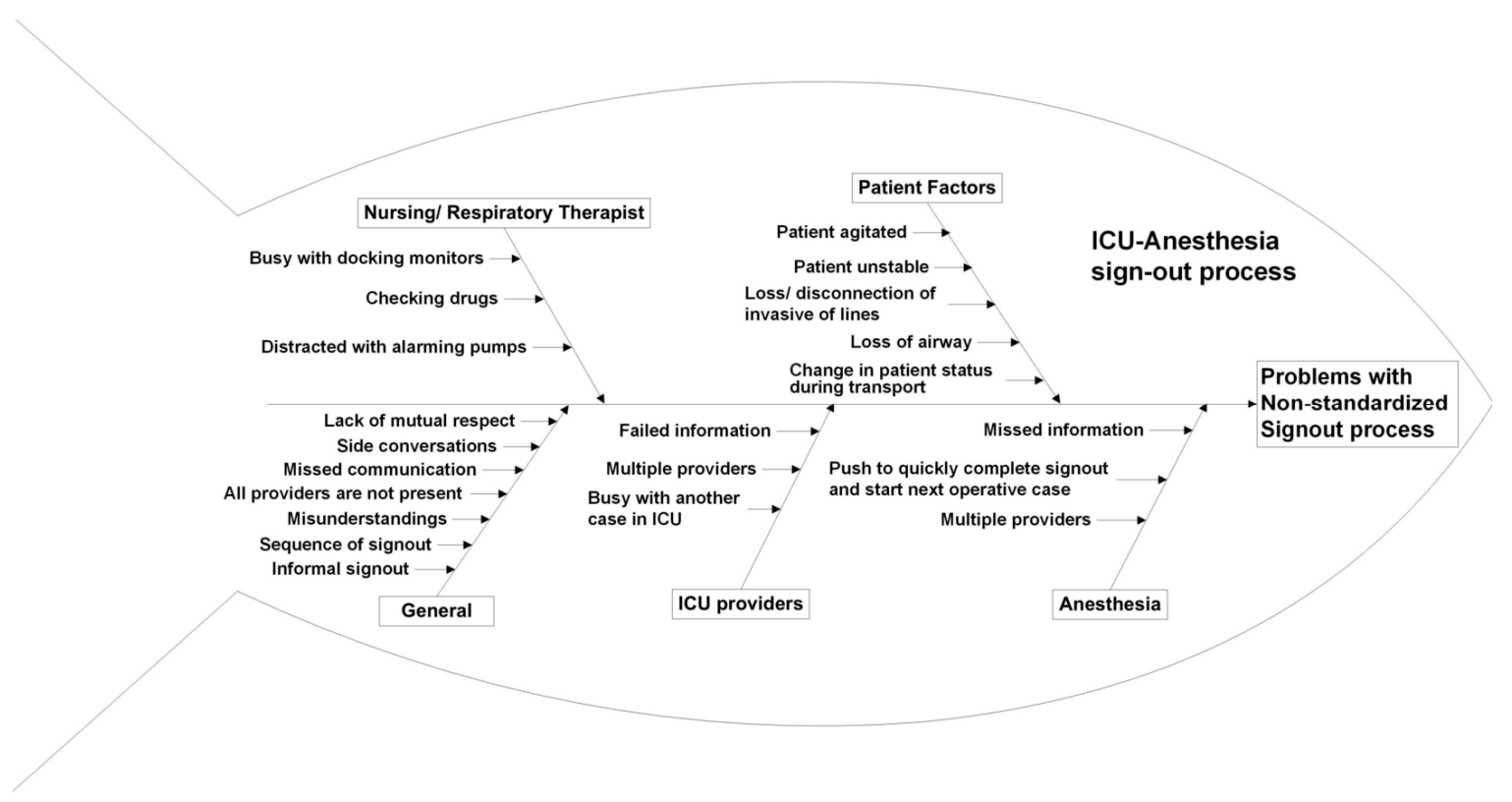

Figure 1. Fish bone diagram showing the causes of the problems contributing to an inefficient/ineffective hand-off process between the anesthesia and operating room teams.

It was identified that a structured hand-off process was needed in order to improve communication amongst staff members and providers. This was designated as the TIME OUT FOR SIGN OUT (TOSO) process based on the Joint Commission "time-out model" as the universal protocol for the start of a procedure [1].

This process entailed a formal interactive sign out between teams, in a standardized sequence, after ensuring that the patient was stable and all members of the multidisciplinary team were able to participate. TOSO was applied to both the entry into and exit from ICUs for all children transported by an anesthesia team. Posters were designed as a prompt to help facilitate the interactive processes (Figure 2A,B). Additionally, a write-on paper tool (prompt form) including important clinical details to be communicated (filled out by the provider initiating the hand-off) was designed to assist providers during the process [7]. This has been described elsewhere [7].

Please see the Appendix A for the prompt form used during the study.

\subsection{Assessing the Intervention}

We performed staff satisfaction surveys and performance audits of our process in order to monitor and evaluate our intervention during the timeline from November 2015 to February 2019. Our QI project was geared towards the deficiencies and inconsistencies with the prior hand-off process between the ICUs and ORs. The pre-implementation survey was used to identify issues we needed to improve as well as provide a marker for satisfaction and improvement in our quality project. The post-implementation surveys identified whether providers felt the process had enhanced patient hand-offs. The performance audit was geared towards identifying the process of the hand-off and whether any critical elements were missed. 


\begin{tabular}{|l|l|l|}
\hline \multicolumn{2}{|l|}{ EXIT TOSO Team: Anesthesiology provider, RN, IRespiratory Therapist, PICU/NICU provider } \\
\hline \multicolumn{3}{|c|}{ TIMtensive Care Unit Transfer of Care to the Perioperative Team } \\
(PICU/NICU to OR “EXIT")
\end{tabular}

(A)

ENTRY TOSO Team: Anesthesiology provider, RN, Surgery provider, \pm Respiratory Therapist, PICU/NICU provider

\begin{tabular}{|l|l|l|}
\hline \multicolumn{3}{|c|}{ TIME OUT FOR SIGN OUT } \\
\hline \multicolumn{1}{|c|}{ Perioperative Transfer of Care to the Intensive Care Unit Team } \\
(OR to PICU/NICU “ENTRY")
\end{tabular}

(B)

Figure 2. (A). Exit intensive care unit (ICU), i.e., hand-off from ICU teams to anesthesia team when transporting child to the OR. (B). Entry ICU, i.e., hand-off from anesthesia and surgery teams to ICU teams when transporting child from operating room (OR) to ICU. 


\subsubsection{Survey}

In order to evaluate the impact of our intervention on staff satisfaction, we conducted an online survey, using SurveyMonkey (Copyright ${ }^{\circledR} 1999-2019$ SurveyMonkey), open to all providers (anesthesia, surgery, nursing, intensivist, and respiratory therapist teams) involved in the care of children during the hand-off process in both NICUs and PICUs. The survey consisted of eight questions that included general data including provider roles, workplace unit and years of experience, as well as pertinent questions about the process. For survey Questions 4, 5, 6 and 7, a qualitative scale of strongly agree, agree, neutral, disagree, strongly disagree was used. The survey was conducted in three phases: pre-implementation/Survey 1 (November-December 2015), six-month post-implementation/Survey 2 (July-August 2016) and $>2$ year follow-up post-implementation/Survey 3 (June-July 2018).

The survey questions are listed below:

(1) What Unit do you work in?

(2) What is your role?

(3) How many years have you worked in your current unit?

(4) The time out for sign out process provides the necessary information about the patient.

(5) The sign out system makes it comfortable for you to ask question to the OR/ICU team members.

(6) The sign out system is efficient and limits needless information about patient care.

(7) At the end of the time out for sign out, do you feel like the sign out was a necessary and important part of patient care?

(8) Do you feel the time out for sign out can be improved?

\subsubsection{Audit}

In order to evaluate the TOSO performance, the clinical head nurse for the pediatric or neonatal ICU silently observed the interaction of the hand-off process during the pre-implementation and post implementation phases. The number of encounters audited was based on the availability of the clinical head nurse (random convenience sample). This was also conducted in three phases-pre-implementation/Audit 1 (November-December 2015), six-month post-implementation/Audit 2 (post-implementation January-June 2016) and $>2$ year follow-up post-implementation/Audit 3 (November 2018-February 2019). The data collected from the audit included: (1) whether a hand-off was completed, (2) critical items covered as part of the hand-off and (3) the hand-off time (time in minutes from the verbalization of initiation of hand-off to the verbalization of completion and dispersion of the team). Based on an expert consensus, the six items that were considered important for discussion at the hand-off included: airway/ventilation, intravenous access, medications administered, pertinent laboratory values, pertinent pre-operative (e.g., previous failed intubation, failed extubation) and pertinent intraoperative events (issues with ventilation intraoperatively, problems with intubation, need for a vasopressor, etc.).

\subsection{Statistical Analysis}

Sigma plot software (SigmaPlot ${ }^{\circledR} 12.5$ Systat Software Inc., San Jose, CA, USA) was used for analysis of statistical data. The general data were expressed as numbers and percentages. The Chi square test was used to test the significance of data on proportion. The data for items missed and number of providers at hand-off process were analyzed using the Kruskal-Wallis one-way analysis of variance on ranks. A $p$ value $<0.05$ was considered significant.

\section{Results}

\subsection{Survey Results}

Overall, the numbers of responses received were as follows: pre-implementation phase, 113 participants; six-month post-implementation survey, 85 participants, and $>2$ year post implementation survey, 135 participants. 
The distribution of the results for individual survey questions 1-3 are as shown in Table 1.

Table 1. Demographic data for provider responses to survey questions 1, 2 and 3, NICU = Neonatal Intensive Care Unit, PICU = Pediatric Intensive Care Unit, CRNA = Certified Registered Nurse Anesthetist, $\mathrm{CRNP}=$ Certified Registered Nurse Practitioner, PA = Physician Assistant. Results are expressed as percentages, values are rounded up.

\begin{tabular}{cccccc}
\hline Survey Questions & Answer Options & $\begin{array}{c}\text { Survey 1 } \\
\text { Response \% } \\
\boldsymbol{n}=\mathbf{1 1 3}\end{array}$ & $\begin{array}{c}\text { Survey 2 } \\
\text { Response \% } \\
\boldsymbol{n}=\mathbf{8 5}\end{array}$ & $\begin{array}{c}\text { Survey 3 } \\
\text { Response \% } \\
\boldsymbol{n}=\mathbf{1 3 5}\end{array}$ & $\begin{array}{c}\boldsymbol{p} \text {-Value, } \\
\text { Chi-Square Test }\end{array}$ \\
\hline Question 1, & Anesthesia & 27 & 29 & 40 & \\
Unit of the Provider & NICU & 38 & 38 & 28 & $p=0.258$ \\
& Pediatric Surgery & 3 & 1 & 4 & \\
\hline PICU & 32 & 32 & 28 & \\
Question 2, & Nurse & 40 & 53 & 23 & \\
Provider role & CRNP/PA & 6 & 7 & 8 & \\
& Attending Physician & 21 & 22 & 23 & \\
& CRNA & 4 & 1 & 11 & \\
Resident/Fellow & 16 & 17 & 22 & \\
Question 3, & Respiratory Therapist & 13 & 0 & 13 & \\
& $<2$ years & 19 & 29 & 26 & \\
\hline
\end{tabular}

The results of survey questions 4-7 are as shown as supporting material in Supplementary Figures S1-S4, respectively. A higher number of anesthesia providers participated in Survey 3 compared to Survey 1. A smaller proportion of participants had more than four years of experience in Survey 3 $(43.2 \%)$ when compared to Survey $1(51.3 \%)$ or Survey $2(50 \%)$; however, this did not achieve statistical significance. There was an increase in the proportion of participants who either strongly agreed or agreed that the process had improved in providing necessary information (Question 4), making it comfortable to ask questions (Question 5), enhancing efficiency (Question 6) and was an important and necessary part of the hand-off process (Question 7), when comparing Survey 1 to Survey 3 (Table 2). In Survey 3, $49.2 \%$ of participants felt that the hand-off process could be improved versus $78.4 \%$ in Survey 1 and $54.2 \%$ in Survey $2(p<0.001)$.

Table 2. Percentage of participants who agreed or strongly agreed.

\begin{tabular}{ccccc}
\hline Survey Question & $\begin{array}{c}\text { Survey 1 } \\
\boldsymbol{n}=\mathbf{1 1 3}\end{array}$ & $\begin{array}{c}\text { Survey 2 } \\
\boldsymbol{n}=\mathbf{8 5}\end{array}$ & $\begin{array}{c}\text { Survey 3 } \\
\boldsymbol{n}=\mathbf{1 3 5}\end{array}$ & $\begin{array}{c}\boldsymbol{p} \text {-Value, } \\
\text { Chi Square Test }\end{array}$ \\
\hline Question 4 (Provides Necessary Information), \% & 40.5 & 90.5 & 89.5 & $<0.001$ \\
Question 5 (Comfortable to Ask Questions), \% & 47.8 & 75.3 & 83.7 & $<0.001$ \\
Question 6 (Efficient and Limits Needless Information), \% & 30.4 & 64.7 & 70.6 & $<0.001$ \\
Question 7 (Necessary and Important), \% & 65.1 & 79.7 & 80.7 & 0.237 \\
\hline
\end{tabular}

Pareto charts of the comments from participants for the three surveys are shown in Figures 3-5, respectively. There was a decrease in the proportion of provider comments with regards to the need for a formal hand-off process after implementation. There was an increase in comments related to expanding the project to other areas including the post-anesthesia care unit and the intermediate care unit following implementation. 


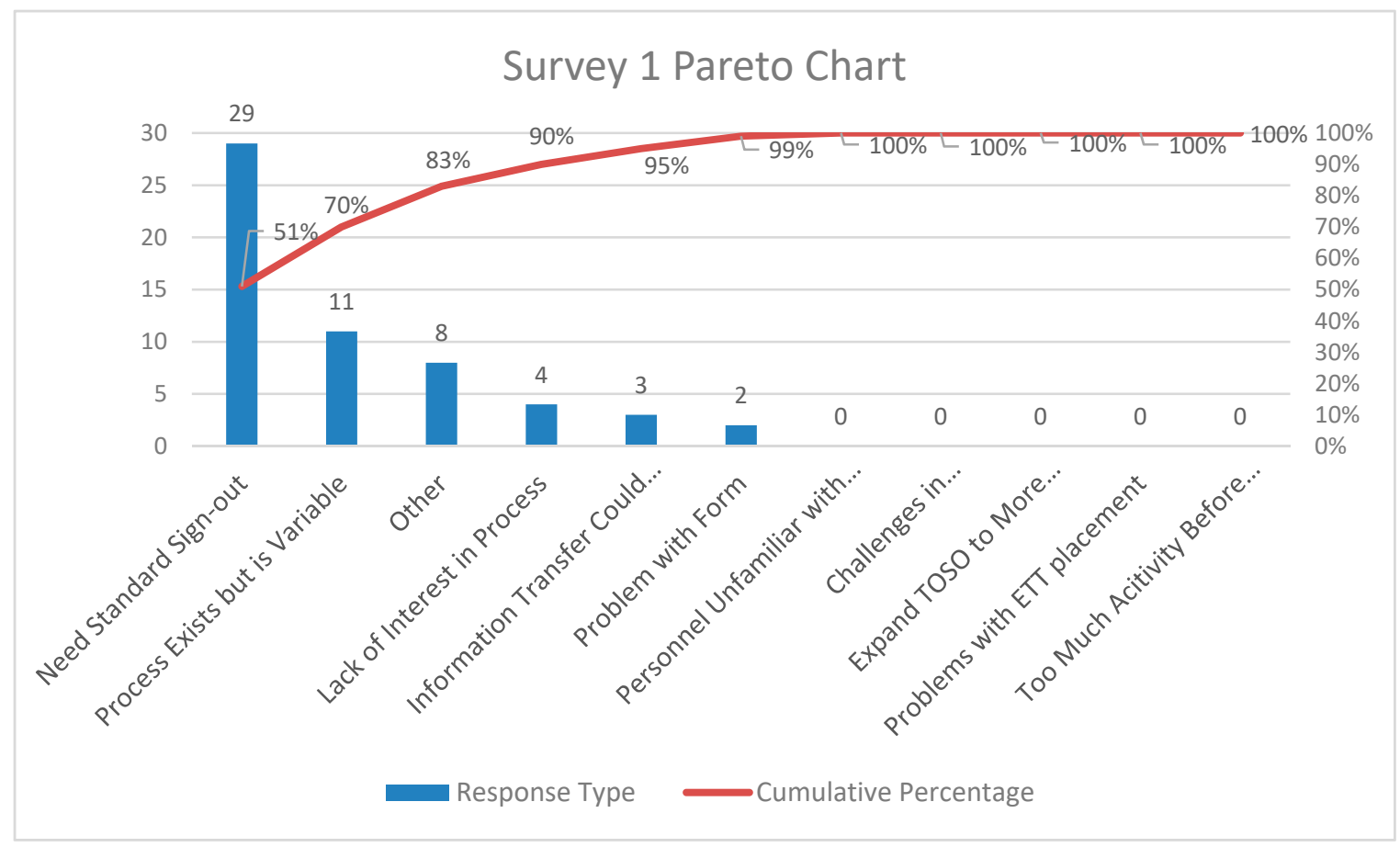

Figure 3. Pareto chart for comments from providers-Survey 1(pre-implementation survey).

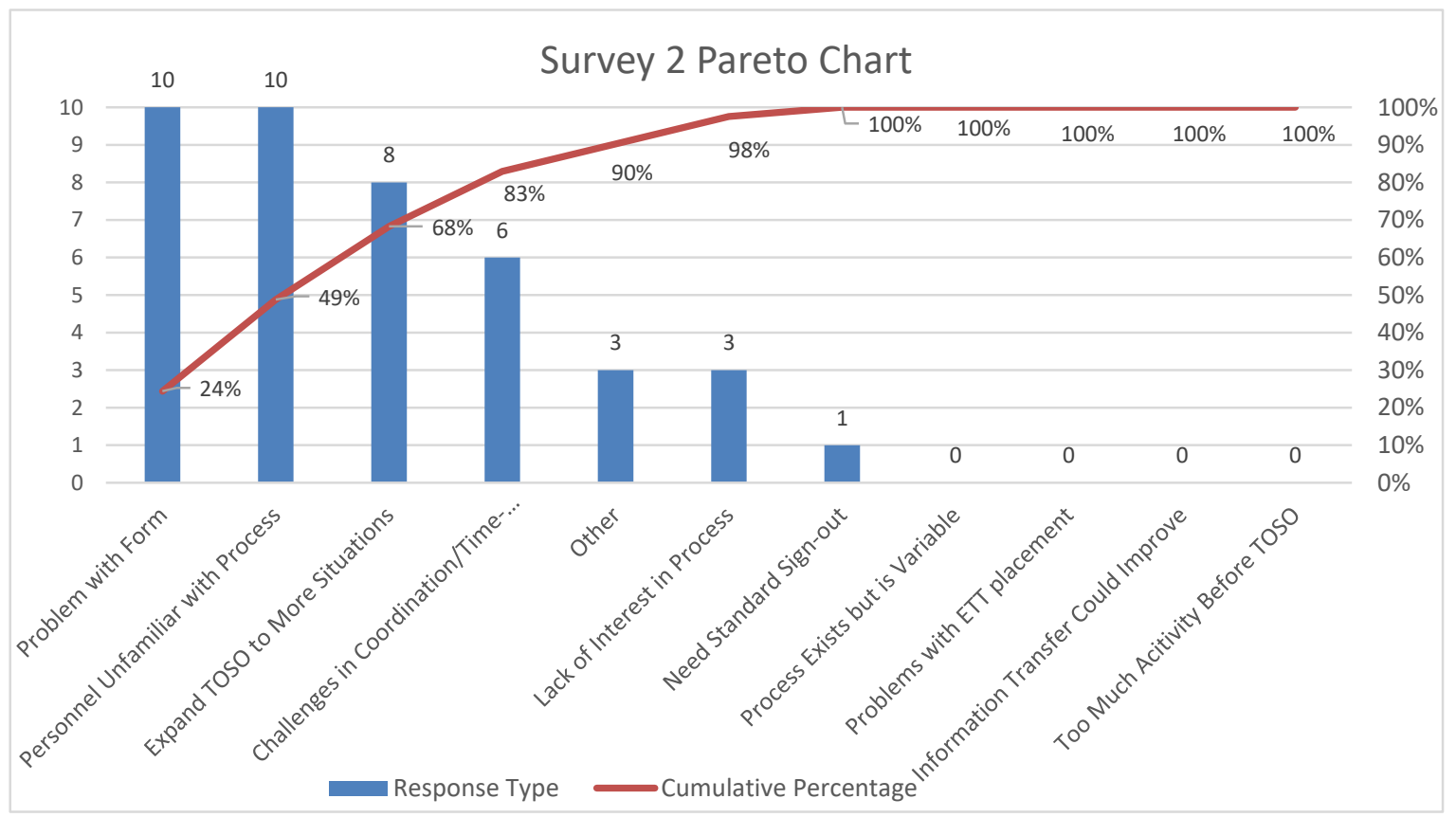

Figure 4. Pareto chart for comments from providers-Survey 2 (six-month post-implementation survey). 


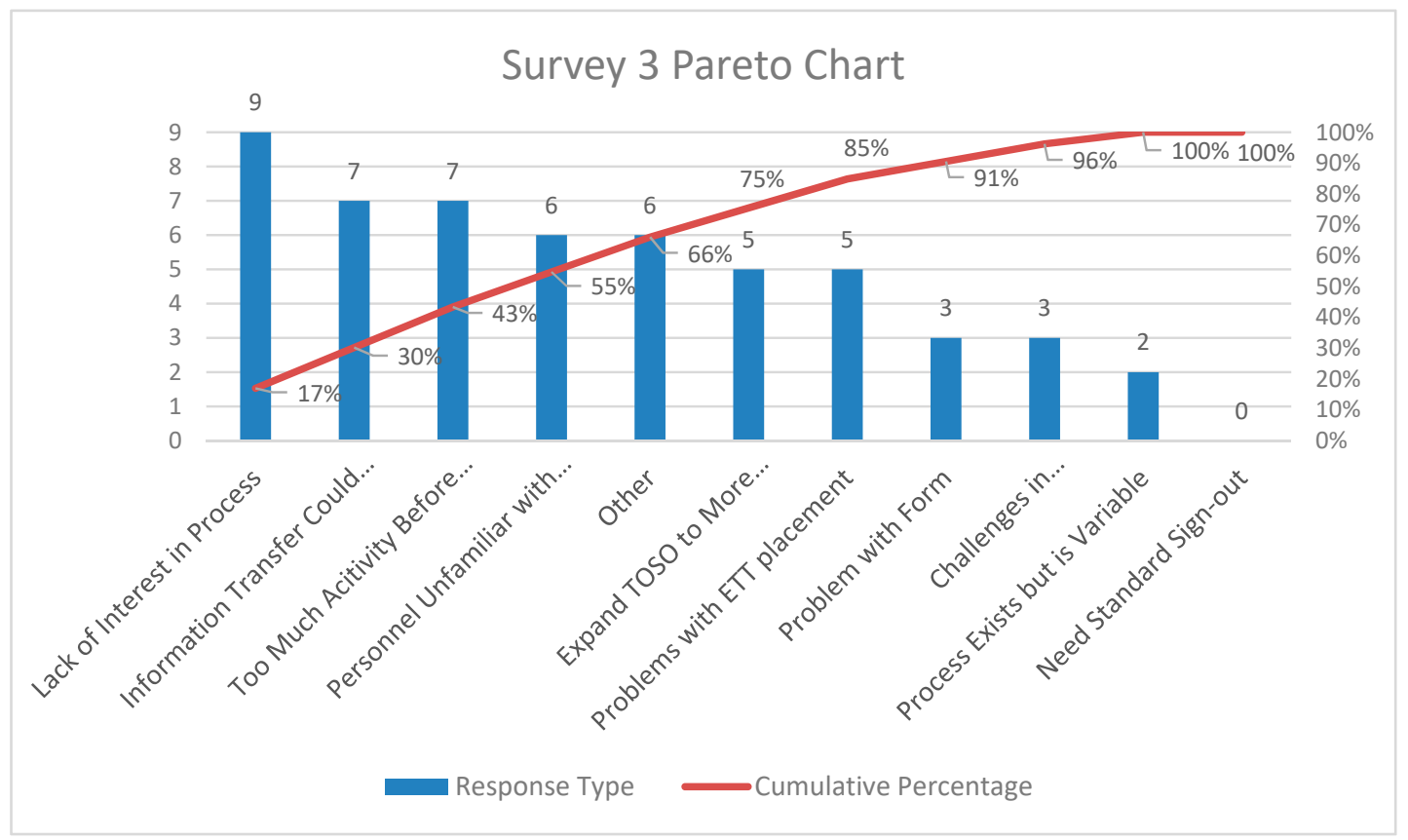

Figure 5. Pareto chart for comments from providers-Survey 3 ( $>2$ years post-implementation survey).

\subsection{Audit Results}

Overall, the number of hand-offs audited were 27 for Audit 1 (pre-implementation), 55 for Audit 2 (6 months post-implementation) and 38 for Audit 3 ( $>2$ years post-implementation). The results of the three audits are as shown in Table 3.

Table 3. Results of the audit regarding missed information at hand-off, IQR = Interquartile range.

\begin{tabular}{|c|c|c|c|c|}
\hline & $\begin{array}{c}\text { Audit 1 } \\
\text { (Pre-Implementation) } \\
n=27\end{array}$ & $\begin{array}{c}\text { Audit } 2 \\
\text { (Six Months Post-Implementation) } \\
n=55\end{array}$ & $\begin{array}{c}\text { Audit } 3 \\
\text { (>2 Years Post-Implementation) } \\
n=38\end{array}$ & $\begin{array}{c}p \text {-Value, } \\
\text { Chi-Square Test }\end{array}$ \\
\hline $\begin{array}{l}\text { Formal Hand-off } \\
\text { Process Occurred, \% }\end{array}$ & 88.8 & 91 & 94.7 & 0.08 \\
\hline $\begin{array}{c}\text { Full Completion of } \\
\text { Hand-off, } \%\end{array}$ & 44.4 & 87.2 & 81.5 & $<0.001$ \\
\hline $\begin{array}{l}\text { Items Missed during } \\
\text { Hand-off, } \%\end{array}$ & 22 & 9 & 5 & $<0.001$ \\
\hline $\begin{array}{c}\text { Laboratory Values } \\
\text { Information Missed, } \\
n(\%)\end{array}$ & $10(37)$ & 7 (12.5) & $6(15.7)$ & 0.032 \\
\hline $\begin{array}{l}\text { Airway Ventilation } \\
\text { Information Missed }\end{array}$ & $5(18.5)$ & $5(8.9)$ & $1(2.6)$ & 0.09 \\
\hline $\begin{array}{c}\text { Medication } \\
\text { Information Missed }\end{array}$ & $5(18.5)$ & $5(8.9)$ & $3(7.8)$ & 0.339 \\
\hline $\begin{array}{l}\text { Time for Completion } \\
\text { of Hand-off, Median, } \\
\text { IQR; Minutes }\end{array}$ & $5(3,8)$ & $5(3.75,7)$ & $6(5,9.75)$ & 0.251 \\
\hline
\end{tabular}

The process timelines for the entire quality improvement project are shown in the supporting information additional figures-Supplementary Figure S5.

\section{Discussion}

Transfer of a patient between the OR and ICU entails two phases: a hand-off process and a physical transfer. The hand-off process is an important part of patient care because an inadequate hand-off can compromise patient safety. The Joint Commission recommends five components to be included in a hand-off. These are represented by the acronym "SHARE": standardize, hardwired, ask questions, reinforce (quality), and educate [8]. The principles of an ideal hand-off process are: 
(1) a real-time face-to-face process, (2) patient-specific, (3) standardized, (4) structured, (5) interactive, (6) conducted with minimal interruption, (7) communicate patient updates, (8) opportunity to ask questions, (9) anticipated plan and (10) ability to easily contact the team signing out following dispersion after hand-off [9]. We have applied these principles by implementing an interactive hand-off process or time out for sign out (TOSO).

In the pre-implementation phase, we identified several problems including the lack of a standardized process, missed information, gaps in communication, as well as limitations with follow-up communication and information ownership. Following detailed meetings of stake holders, literature searches, and identifying the above problems, we standardized our hand-off process to address these concerns. A key component of our TOSO was the interaction between the teams to ensure a detailed hand-off and transfer of responsibility. Further, our interactive process ensured that (1) the hand-off process commenced only after ensuring the stability of the patient, (2) all participants were present during the process, and (3) there was clear verbalization of the transfer/acceptance of responsibility.

We followed our pre-implementation baseline survey with a post-implementation survey at 6 months after implementation, and another survey $>2$ years after implementation. Overall, staff felt there was an improvement in the transfer of information, comfort in asking questions, and efficiency and importance of the hand-off process following the institution of a formal process. Our surveys were useful in assessing our process change from the individual provider's perspective. We followed up with an additional post-implementation survey to evaluate the continued success of our QI process. Across the three surveys, there was a decrease in the number of providers who felt that the process could be improved. Based on provider comments, identified areas for improvement included expanding the hand-off process to other areas such as the post-anesthesia care unit or intermediate care unit.

Following the successful implementation of our quality project in children's ICUs, our TOSO process for "Exit" and "Entry" ICU has been expanded to adult ICUs throughout the hospital and designated the "universal Time out for sign out process" [7]. Although information on the hand-off tool for each ICU may differ, we endeavored to have pertinent information on the hand-out paper tool, with a minimal risk of losing important information [7]. Our emphasis was on the "interactive" processes of communication rather than a paper tool which can be available for later referral by the ICU team. Although a universal TOSO for all ICUs, including adult ICUs, appears to be appropriate and useful, there is a risk of losing specific pediatric-related information. Perhaps a general adaptability of the paper tool may not be feasible, although the principles of TOSO are still applicable [7].

The model that we used for our standardized process is similar to that reported by other quality investigators in the ICU setting [3]. We involved stake holders from relevant disciplines who were responsible for disseminating the initiation, project progression, implementation, staff education and communication for their respective disciplines. In contrast to another study [3], we have demonstrated a significant improvement in each of the survey question items (4,5 and 6; provides necessary information, comfortable to ask questions and efficient). Further, our survey results represent multidisciplinary providers and includes a comprehensive team of physicians (anesthesia, intensivist, and surgeon), nurses and respiratory therapists (not included in other studies).

A previous study [10] utilized expertise from Formula 1 and aviation industries to improve patient handover in the operating room-ICU setting in a children's hospital. The investigators noted that a great variability in the health care setup work force has potential for missed information, miscommunication, staff dissatisfaction and unfavorable outcomes.

A structured handover process for pediatric cardiac surgery has been described, involving a pre-handover form filled out by an OR nurse and sent to the pediatric ICU via a pneumatic pod system, allowing staff to anticipate and be appropriately ready when they receive the patient [11]. The authors found an improvement in transferring the information of 19 essential points [11]. This finding is similar to our study where we observed a decrease in the missed critical items at the time of hand-off, following the implementation of the standardized process. We focused on the interactivity of our standardized hand-off process, reiterating the value of communication, leadership and teamwork, considered as nontechnical 
skills contributing significantly to human errors [12-14]. Our standardized process incorporates all these aspects of a hand-off that begins with the pre hand-off (nurse calls the ICU for a hand-off over phone informing the current status) followed by an interactive standardized hand-off when all parties are present, the patient is stable, monitors are docked and there are no distractions. Our longitudinal analysis demonstrates the continued staff satisfaction and improvement while identifying areas for further improvement. This sustainability is in keeping with another study that demonstrated the continued efficacy of a standardized handover in a pediatric cardiac ICU [15].

Our study reports an improved staff satisfaction in the post-implementation phase similar to a previous study [3] that reported an improved satisfaction of staff and clarity regarding the start and end of handover process [3]. The TOSO was at the cost of no additional time, yet was a succinct process.

\section{Study Limitations}

The survey relied on self-reporting by the providers. There was observer bias with regard to conducted audits. The number of encounters audited were based on the availability of the auditor (clinical head nurse). The inter-rater reliability of the auditors was not tested. A standardized audit process could have reduced observer variability and bias. However, this needs more resources and warrants future studies in this direction. Further, we have not studied the interactions between providers' professional and mutual respect at hand-off. This would warrant detailed research with standardized protocols and video clips to assess interactions. Ours was a QI project aimed at standardizing and improving our hand-off process. Improved patient outcomes, reductions in morbidity and decreases in missed information rates would be more suitable markers to judge the efficacy and success of the standardized interactive hand-off process. Although, improved outcomes have been reported in one study [16], these outcome measures have several confounding variables not taking into account patient comorbidities, observer bias and other associated causes.

\section{Conclusions}

The results of our QI project demonstrate an overall improved efficiency, satisfaction and sustainability of the standardized interactive hand-off process for the transfer of care between children's ICUs and operating rooms. Perhaps it may serve as a model for other institutions.

Supplementary Materials: The following are available online at http://www.mdpi.com/2227-9067/7/9/123/s1, Figure S1: Distribution of responses for Survey Question 4-the time out for sign out process provides the necessary information about the patient. Figure S2: Distribution of responses for Survey Question 5-the sign out system makes it comfortable for you to ask question to the OR/ICU team members. Figure S3: Distribution of responses for Survey Question 6- the sign out system is efficient and limits needless information about patient care. Figure S4: Distribution of responses for Survey Question 7-at the end of the time out for sign out, do you feel like the sign out was a necessary and important part of patient care. Figure S5: Timelines for the Quality Improvement (QI).

Author Contributions: P.G.D. conceptionalized, designed and implemented the study, collected and analyzed the data, wrote the manuscript, critically appraised the manuscript; T.J.C. conceptionalized, designed and implemented the study, collected and analyzed the data, helped write the manuscript, critically appraised the manuscript; T.K.M.D. conceptionalized, designed and implemented the study, collected and analyzed the data, helped write the manuscript, critically appraised the manuscript; A.A.P. conceptionalized, designed and implemented the study, critically appraised the manuscript; M.C.W. designed and implemented the study, helped analyze data, critically appraised the manuscript; J.B.C. conceptionalized, designed and implemented the study, critically appraised the manuscript; L.L. helped with data analysis, writing the manuscript and critically appraised the manuscript; D.J.M. conceptionalized, designed and implemented the study, critically appraised the manuscript; R.E.C. conceptionalized, designed and implemented the study, critically appraised the manuscript. All authors have read and agreed to the published version of the manuscript.

Funding: This research received no external funding.

Acknowledgments: We acknowledge the contributions of Diane E McCloskey, Department of Anesthesiology and Perioperative Medicine, for her help with the manuscript and figures, Jocelyn Johnson, Quality Coordinator, Department of Anesthesiology and Perioperative Medicine, for educating nursing staff, Jackie Lamendola, Department of Anesthesiology and Perioperative Medicine, former Quality Program Manager for her tremendous help with initiation of this project, Michele Smith, Debra Rovito, Children's Surgery Program Manager and Jill Arnold, Newborn Medicine Nursing leadership, Penn State Health Hershey Medical Center, Hershey. We also 
thank all the anesthesia providers, nurses, surgeons, intensivists and respiratory therapists for their help and cooperation in making this process successful.

Conflicts of Interest: The authors declare no conflict of interest.

\section{Appendix A}

Exit and Entry ICU (Intensive Care Unit) prompt forms, NICU = Neonatal Intensive Care Unit, $\mathrm{PICU}=$ Pediatric Intensive Care Unit, ETT $=$ Endotracheal tube IV $=$ intravenous, $\mathrm{FiO} 2=$ Fractional inspired oxygen concentration, Paw = Peak airway pressure, TV = tidal volume, PS = pressure support, Hct $=$ hematocrit.

\section{EXIT-ICU: NICU/PICU to Anesthesia Transfer of Care: Time out for Sign out Tool}

(not a part of medical record)
Name
DOB
MR \#
Procedure

1. Comorbidities

2. Access-peripheral

central venous

length

3. ETT/tracheostomy-size

4. Ventilator settings $\mathrm{FiO} 2 \quad$ Mode

5. IV infusions

6. Medications due for Operating room

7. Availability of blood/blood products

8. Last set of labs $\mathrm{Hb} / \mathrm{Hct}$

9. Post-operative plan for ventilation

10. Any issues/concerns

ICU attending, contact \#
Blood glucose

ICU resident contact \#

\section{ENTRY ICU: Anesthesia Team to NICU/PICU Transfer of Care: Time out for Sign out}

(not a part of medical record)

\section{Name DOB}

1. Airway ETT

size

2. Recommended Ventilation settings, $\mathrm{FiO} 2$

3. New access established in OR

4. Anesthetic agents used

5. Last dose of narcotics, anesthetic agent, sedative or neuromuscular blocking agent

6. Analgesics in OR-acetaminophen, narcotics, regional block and infiltration of surgical wound

7. Reversal agents administered

8. If epidural-plan, drug, infusion rate

9. Antibiotics administered in OR, dose and time

10. IV infusions-inotropes, dosage rate

11. Total fluids administered

12. Blood or blood products

13. Intraoperative labs

14. CP bypass,

15. Any specific issues and concerns Attending Anesthesiologist contact \#

MR \#

Procedure

type length issues

Paw TV Ventilation mode PEEP 


\section{References}

1. The Joint Commission. Handsoff Communications: Toolkit for Implementing the National Patient Safety Goal; Joint Commission Resources: Oak Brook, IL, USA, 2008; Volume 65.

2. Horwitz, L.I.; Meredith, T.; Schuur, J.D.; Shah, N.R.; Kulkarni, R.G.; Jenq, G.Y. Dropping the baton: A qualitative analysis of failures during the transition from emergency department to inpatient care. Ann. Emerg. Med. 2009, 53, 701-710.e4. [CrossRef]

3. Krimminger, D.; Sona, C.; Thomas-Horton, E.; Schallom, M. A Multidisciplinary QI Initiative to Improve OR-ICU Handovers. Am. J. Nurs. 2018, 118, 48-59. [CrossRef] [PubMed]

4. Petrovic, M.A.; Aboumatar, H.; Baumgartner, W.A.; Ulatowski, J.A.; Moyer, J.; Chang, T.Y.; Camp, M.S.; Kowalski, J.; Senger, C.M.; Martinez, E.A. Pilot implementation of a perioperative protocol to guide operating room-to-intensive care unit patient handoffs. J. Cardiothorac. Vasc. Anesth. 2012, 26, 11-16. [CrossRef]

5. Pickering, B.W.; Hurley, K.; Marsh, B. Identification of patient information corruption in the intensive care unit: Using a scoring tool to direct quality improvements in handover. Crit. Care Med. 2009, 37, 2905-2912. [CrossRef]

6. Boat, A.C.; Spaeth, J.P. Handoff checklists improve the reliability of patient handoffs in the operating room and postanesthesia care unit. Paediatr. Anaesth. 2013, 23, 647-654. [CrossRef]

7. Karamchandani, K.; Fitzgerald, K.; Carroll, D.; Trauger, M.E.; Ciccocioppo, L.A.; Hess, W.; Prozesky, J.; Armen, S.B. A Multidisciplinary Handoff Process to Standardize the Transfer of Care Between the Intensive Care Unit and the Operating Room. Qual. Manag. Health Care 2018, 27, 215-222. [CrossRef]

8. Joint Commission Center for Transforming Healthcare Releases Targeted Solutions Tool for Hand-Off Communications. Joint Comm. Perspect. 2012, 32. Available online: https://www.jointcommission.org/-/media/de precated-unorganized/imported-assets/tjc/system-folders/blogs/tst_hoc_persp_08_12pdf.pdf?db=web\&hash =BA7C8CDB4910EF6633F013D0BC08CB1C (accessed on 2 September 2020).

9. Petrovic, M.A.; Martinez, E.A.; Aboumatar, H. Implementing a perioperative handoff tool to improve postprocedural patient transfers. Jt. Comm. J. Qual. Patient Saf. 2012, 38, 135-142. [CrossRef]

10. Catchpole, K.R.; de Leval, M.R.; McEwan, A.; Pigott, N.; Elliott, M.J.; McQuillan, A.; MacDonald, C.; Goldman, A.J. Patient handover from surgery to intensive care: Using Formula 1 pit-stop and aviation models to improve safety and quality. Paediatr. Anaesth. 2007, 17, 470-478. [CrossRef] [PubMed]

11. Craig, R.; Moxey, L.; Young, D.; Spenceley, N.S.; Davidson, M.G. Strengthening handover communication in pediatric cardiac intensive care. Paediatr. Anaesth. 2012, 22, 393-399. [CrossRef] [PubMed]

12. Fletcher, G.C.; McGeorge, P.; Flin, R.H.; Glavin, R.J.; Maran, N.J. The role of non-technical skills in anaesthesia: A review of current literature. Br. J. Anaesth. 2002, 88, 418-429. [CrossRef] [PubMed]

13. Donchin, Y.; Gopher, D.; Olin, M.; Badihi, Y.; Biesky, M.; Sprung, C.L.; Pizov, R.; Cotev, S. A look into the nature and causes of human errors in the intensive care unit. 1995. Qual. Saf. Health Care 2003, 12, 143-147. [CrossRef] [PubMed]

14. Horwitz, L.I.; Moin, T.; Krumholz, H.M.; Wang, L.; Bradley, E.H. Consequences of inadequate sign-out for patient care. Arch. Intern. Med. 2008, 168, 1755-1760. [CrossRef] [PubMed]

15. Chenault, K.; Moga, M.A.; Shin, M.; Petersen, E.; Backer, C.; De Oliveira, G.S., Jr.; Suresh, S. Sustainability of protocolized handover of pediatric cardiac surgery patients to the intensive care unit. Paediatr. Anaesth. 2016, 26, 488-494. [CrossRef] [PubMed]

16. Agarwal, H.S.; Saville, B.R.; Slayton, J.M.; Donahue, B.S.; Daves, S.; Christian, K.G.; Bichell, D.P.; Harris, Z.L. Standardized postoperative handover process improves outcomes in the intensive care unit: A model for operational sustainability and improved team performance. Crit. Care Med. 2012, 40, 2109-2115. [CrossRef] [PubMed]

(C) 2020 by the authors. Licensee MDPI, Basel, Switzerland. This article is an open access article distributed under the terms and conditions of the Creative Commons Attribution (CC BY) license (http://creativecommons.org/licenses/by/4.0/). 